\title{
Identification of a rare SEPT9 variant in a family with autosomal dominant Charcot- Marie-Tooth disease
}

\author{
Gerrit M. Grosse ${ }^{1 *}\left(\mathbb{D}\right.$, Christine Bauer $^{2}$, Bruno Kopp $^{1}$, Christoph Schrader $^{1+}$ and Alma Osmanovic ${ }^{1 \dagger}$
}

\begin{abstract}
Background: Charcot-Marie-Tooth disease (CMT) is one of the most commonly inherited neurological disorders. A growing number of genes, involved in glial and neuronal functions, have been associated with different subtypes of CMT leading to improved diagnostics and understanding of pathophysiological mechanisms. However, some patients and families remain genetically unsolved.

Methods: We report on a German family including four affected members over three generations with a CMT phenotype accompanied by cognitive deficits, predominantly with regard to visual abilities and episodic memory.

Results: A comprehensive clinical characterization followed by a sequential diagnostic approach disclosed a heterozygous rare SEPT9 missense variant c.1406 T > C, p.(Val469Ala), that segregates with disease. SEPT9 has been linked to various intracellular functions, such as cytokinesis and membrane trafficking. Interestingly, SEPT9-mutations are known to cause hereditary neuralgic amyotrophy (HNA), a recurrent focal peripheral neuropathy.
\end{abstract}

Conclusion: We, for the first time, present a SEPT9 variant associated to a CMT phenotype and suggest SEPT9 as new sufficient candidate gene in CMT.

Keywords: Charcot-Marie-Tooth, Next generation sequencing, SEPT9, Septin, Inherited neuropathy

\section{Background}

Charcot-Marie-Tooth disease (CMT), also known as hereditary motor and sensory neuropathy, comprises a genetically and clinically heterogeneous group of genetic disorders that mainly affect peripheral nerves. CMT is clinically characterized by distal muscle weakness and atrophy, sensory loss, depressed tendon reflexes, and foot deformity. Growing knowledge about additional clinical features associated with CMT makes it a syndromic disease [1]. Most commonly, CMT is neurophysiologically subdivided into a demyelinating (CMT1) and axonal (CMT2) form depending on whether nerve

\footnotetext{
* Correspondence: grosse.gerrit@mh-hannover.de

${ }^{+}$Christoph Schrader and Alma Osmanovic contributed equally to this work. 'Department of Neurology, Hannover Medical School, Carl-Neuberg-Str. 1, 30625 Hannover, Germany

Full list of author information is available at the end of the article
}

conduction velocity (NCV) is below or above $38 \mathrm{~m} / \mathrm{s}[2]$. Up to $60-70 \%$ of demyelinating CMT cases are caused by duplication of peripheral myelin protein 22 gene (PMP22). Together with mutations in myelin protein zero (MPZ), gap junction protein beta 1 (GJB1), and mitofusin 2 (MFN2), over half of CMT cases can be explained [3]. So far, more than $80 \mathrm{CMT}$-genes have been identified mainly through linkage analysis and next generation sequencing (NGS) studies. Most of the identified genes related to CMT are encoded in nerval structures like axons or myelin and functionally involved in cytoskeleton network, membrane trafficking and myelination. Therefore, genes involved in cellular processes and biological functions concerning Schwann cells and axonal transport processes, are of high interest in so far genetically unsolved CMT patients [4]. 
In this study we performed a sequential approach for genetic testing, including a NGS multi gene panel with known CMT-genes and promising candidate genes, in a clinically well characterised CMT1-family. We identified a rare heterozygous missense variant in the SEPT9 gene, a gene previously described as a cause of hereditary neuralgic amyotrophy (HNA) when heterogeneously mutated [5]. SEPT9 is part of the SEPT3-group and encodes the ubiquitously expressed septin 9 protein, which belongs to the conserved septin family of GTPases involved in various cellular processes such as motility and cytokinesis [6]. Interestingly, septin 9 interacts with the cytoskeleton including microtubules and actin and thereby promotes asymmetric neurite outgrowth [7]. Here, we genetically and clinically present an association of a SEPT9 gene alteration to a distinct CMT phenotype and discuss potential effects on protein level.

\section{Methods}

\section{Subjects}

We investigated a northern German CMT1-family with four affected members over three generations. The local ethics committee and all participants consented to the analysis. Three individuals could be enrolled (Fig. 1a).

Index case II.3 underwent an extensive work-up including a thorough clinical evaluation, physical examination, nerve conduction studies, cranial magnetic resonance imaging (MRI), lumbar puncture, routine and specified blood tests. Cognition was assessed using the Montreal Cognitive Assessment (MOCA) [8] and additionally using the "Consortium to Establish a Registry for Alzheimer's Disease" battery (CERADplus) [9].

Healthy subject III.2 and his affected brother III.4 were evaluated subsequently by neurological examinations, nerve conduction studies and neuropsychological testing, as described above. Individual III.4 was further assessed by cranial MRI.

\section{Genetic testing}

Deletions and Duplications in PMP22, MFN2 and MPZ were analysed by Multiplex ligation-dependent probe amplification (MLPA), performed on extracted DNA obtained from index patient II.3 whole blood sample.

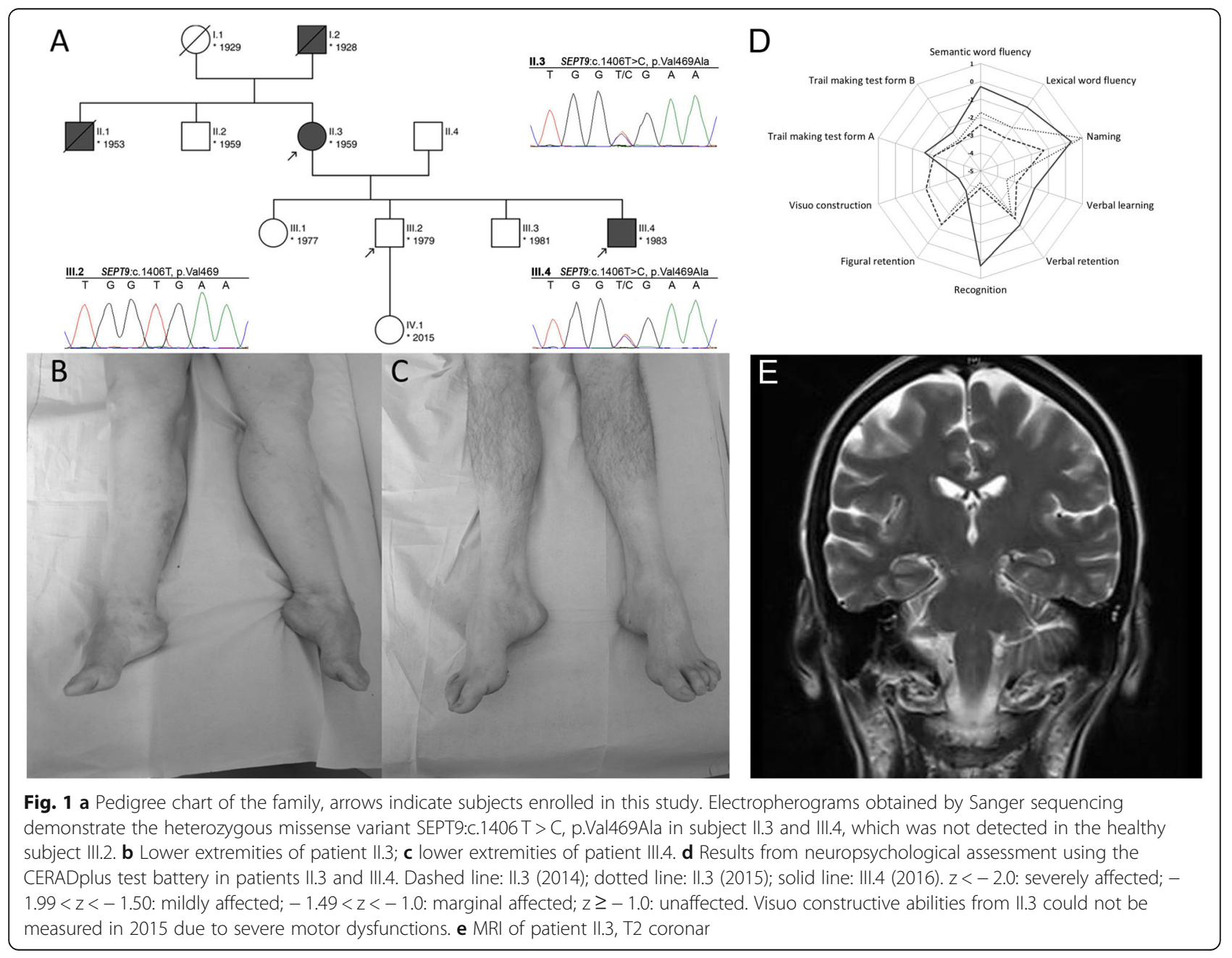


Subsequently, NGS was carried out in this subject at the CeGat-Institute, Tübingen, Germany, with a multi gene panel-based strategy including following genes known to be associated with or linked to hereditary neuropathy: AARS, ABHD12, AIFM1, ARHGEF10, ATL1, ATL3, BSCL2, C10ORF2, C12ORF65, CTDP1, DCAF8, DHTKD1, DNM2, DNMT1, DST, DYNC1H1, EGR2, FAM134B, FBLN5, FGD4, FIG4, GAN, GARS, GDAP1, GJB1, GNB4, HADHA, HADHB, HARS, HINT1, HK1, HOXD10, HSPB1, HSPB8, IFRD1, IGHMBP2, IKBKAP, INF2, KARS, KIF1A, KIF1B, KIF5A, LITAF, LMNA, LRSAM1, MARS, MED25, MFN2, MPZ, MTMR14, MTMR2, NDRG1, NEFL, NGF, NTRK1, OPA1, OPA2, PDHA1, PDK3, PLEKHG5, PMP22, POLG, PRPS1, PRX, RAB7A, REEP1, SBF1, SBF2, SCN9A, SEPT9, SH3TC2, SLC12A6, SOX10, SPTLC1, SPTLC2, SURF1, TFG, TRIM2, TRPV4, TTR, TYMP, WNK1, YARS. Only variants affecting coding exons or canonical splice sites were selected. Synonymous variants were filtered out. Since the CMT1-family in our study expose an autosomal dominant inheritance pattern only rare variants with a minor allele frequency (MAF) of $<0.1 \%$ in the European population, according to the global databases, ExAC Browser (http://exac.broadinstitute.org/) and gnomAD (https://gnomad.broadinstitute.org/) as well as an inhouse database of the CeGat Institute, Tübingen, Germany, were considered. In silico predictions of the longest SEPT9 transcript ENST00000427177.6 (NM_ 006640) were taken from Alamut Visual software version $2.8^{\circ}$ (Interactive Biosoftware) which combines different prediction programs (SIFT, Mutation Taster and PolyPhen) (Table 1). For further variant characterisation the Combined Annotation Dependent Depletion (CADD) score [11] and the American College of Medical Genetics (ACMG) criteria [10] were used. We verified and evaluated our identified variant for co-segregation with the phenotype in individuals III.2 and III.4 by Sanger sequencing (Fig. 1a).

\section{Results}

\section{Clinical features}

The index patient II.3, a then 55-years-old woman with a past medical history of breast cancer and B-cell non-
Hodgkin lymphoma, presented with progressive muscular weakness and moderate sensory deficits, with an estimated symptom onset at 40 years. Neurological examination revealed bilateral pes cavus (Fig. 1b), intermittent postural tremor of the right upper extremity, distal muscle weakness, diminished deep tendon reflexes and hypesthesia of the lower extremities as well as severe gait ataxia. Furthermore, she complained of forgetfulness, impaired concentration and disorientation, first noted at the age of 53 years. Her nerve conduction studies were consistent with a primarily demyelinating motor and sensory polyneuropathy (Table 2). The initial neuropsychological assessment of II.3 yielded a MOCA score of 20/30, indicating the presence of cognitive deficits. The more comprehensive assessment with the CERADplus neuropsychological test battery revealed deficits mainly in visual constructive abilities $(z=-1.8)$, in verbal learning and memory (verbal learning, $z=-2.88$; verbal recognition, $z=-4.05)$, and on both Trail Making Tests (Form A, $z=-2.25$; Form B, $z=-3.01$ ) (see also Fig. 1d; dashed line). Her estimated premorbid intelligence, as determined using a multiple-choice vocabulary intelligence test (MWT-B) [12] (raw score: 21), fell within the range of age-adjusted normal values, rendering it unlikely that the observed cognitive deficits were due to premorbid mental retardation. Cranial MRI revealed neither general nor regional atrophy nor white matter lesions (Fig. 1e). Blood tests and cerebrospinal fluid analysis did not show any pathological results, including normal results for Tau, pTau and Amyloid- $\beta$.

At follow-up 2 years later, the patient was dependent of walking aids and of legal guardianship for financial affairs. The initially observed pattern of cognitive deficits proved remarkably stable (see Fig. 1d; dotted line).

A second family subject, II.3 youngest son (III.4, *1983), exhibit almost similar symptoms: bilateral pes cavus (Fig. 1c), intermittent postural tremor of the left upper extremity, hypesthesia of the lower extremities and severe gait ataxia, while demonstrating an earlier age at onset approximately with 20 years, and a more severe primarily demyelinating sensory and motor neuropathy in nerve conduction studies (Table 2). Neuropsychological assessment of III.4 yielded a MOCA

Table 1 Rare heterozygous non-silent variant in the SEPT9 gene predicted to be deleterious identified in a german family with CMT

\begin{tabular}{|c|c|c|c|c|c|c|c|c|c|c|}
\hline \multirow[t]{2}{*}{ Sample } & \multirow{2}{*}{$\begin{array}{l}\text { Chromosomal } \\
\text { position } \\
\text { (GRCh37/ } \\
\text { hg19) }\end{array}$} & \multirow[t]{2}{*}{ Exon } & \multirow{2}{*}{$\begin{array}{l}\text { Nucleotide } \\
\text { change }\end{array}$} & \multirow{2}{*}{$\begin{array}{l}\text { Amino-acid } \\
\text { change }\end{array}$} & \multirow{2}{*}{$\begin{array}{l}\text { SNP } \\
\text { number }\end{array}$} & \multirow[t]{2}{*}{ MAF } & \multicolumn{3}{|l|}{ Prediction } & \multirow[t]{2}{*}{ ACMG } \\
\hline & & & & & & & SIFT & PolyPhen-2 & Mutation-Taster & \\
\hline $\begin{array}{l}1.3 \\
111.4\end{array}$ & $17: 75488782$ & 8 & c. $1406 \mathrm{~T}>\mathrm{C}$ & p.(Val469Ala) & rs376712636 & 0.0006837 & Deleterious & Benign & disease causing & Class 3 (VUS) \\
\hline |ll.2 & / & / & / & / & / & / & / & / & / & / \\
\hline
\end{tabular}

Abbreviations: ACMG American College of Medical Genetics and Genomics according to Richards et al. 2015 [10], Genetics in Medcine, MAF minor allele frequency in European (non-Finnish) population according to Exome Aggregation Consortium Browser (Beta); Reference sequence used: NM_006640. VUS variant of uncertain significance 
Table 2 Clinical and diagnostic characteristics of two SEPT9 variant carriers (II.3 and III.4) compared to an unaffected family member (II.2)

\begin{tabular}{|c|c|c|c|c|c|c|c|c|c|c|c|c|c|c|c|c|}
\hline \multirow[t]{3}{*}{ Subject } & \multirow[t]{3}{*}{ Sex } & \multirow{3}{*}{$\begin{array}{l}\text { Genetics } \\
\text { Nucleotide } \\
\text { Change }\end{array}$} & \multicolumn{6}{|c|}{ Clinical features } & \multicolumn{8}{|c|}{ Nerve conduction studies } \\
\hline & & & \multirow{2}{*}{$\begin{array}{l}\text { Age at } \\
\text { Onset } \\
\text { (y) }\end{array}$} & \multirow[t]{2}{*}{$\begin{array}{l}\text { Symptom at } \\
\text { Onset }\end{array}$} & \multirow{2}{*}{$\begin{array}{l}\text { Amyotrophy } \\
\text { UL/LL }\end{array}$} & \multirow{2}{*}{$\begin{array}{l}\text { Weakness } \\
\text { UL/LL }\end{array}$} & \multirow{2}{*}{$\begin{array}{l}\text { Sensory } \\
\text { loss } \\
\text { UL/LL }\end{array}$} & \multirow{2}{*}{$\begin{array}{l}\text { DTR } \\
\text { UL/ } \\
\text { LL }\end{array}$} & \multicolumn{4}{|c|}{ Ulnar nerve } & \multicolumn{2}{|c|}{$\begin{array}{l}\text { Peroneal } \\
\text { nerve }\end{array}$} & \multicolumn{2}{|l|}{$\begin{array}{l}\text { Sural } \\
\text { nerve }\end{array}$} \\
\hline & & & & & & & & & CMAP & $\begin{array}{l}\mathrm{DL} / \\
\mathrm{MNCV}\end{array}$ & SNAP & $\mathrm{SCV}$ & CMAP & $\begin{array}{l}\mathrm{DL} / \\
\text { MNCV }\end{array}$ & SNAP & $\mathrm{SCV}$ \\
\hline $11.3+$ & $\mathrm{F}$ & $\begin{array}{l}\text { SEPT9, } \\
\text { C.1406 T > } \\
\text { C }\end{array}$ & $40^{\#}$ & $\begin{array}{l}\text { ataxia, hyp- } \\
\text { aesthesia }\end{array}$ & $++/++$ & $+/+$ & $+/+$ & $1 / 0$ & 5.3 & $4.6 / 37$ & NR & 31 & 1.3 & $7.2 / 30$ & NR & $\overline{N R}$ \\
\hline$\| 11.2$ & M & - & N/A & N/A & $-/-$ & $-1-$ & $-/-$ & $2 / 2$ & 8.9 & $2.9 / 62$ & 17.8 & 50 & 10.1 & $3.8 / 50$ & 17.8 & 47 \\
\hline |ll.4 & M & $\begin{array}{l}\text { SEPT9, } \\
\text { c.1406 T > } \\
\text { C }\end{array}$ & $20^{\#}$ & $\begin{array}{l}\text { ataxia, hyp- } \\
\text { aesthesia }\end{array}$ & $++/++$ & $-/-$ & $-/+$ & $1 / 1$ & 6.0 & $5.2 / 21$ & NR & 27 & NR & NR & NR & NR \\
\hline
\end{tabular}

Index case (†), M male, F female, LL lower limbs, UL upper limbs; amyotrophy (none, -; slight, +; moderate, ++; severe, +++); weakness (none, -; slight, +; moderate, ++ ; severe, +++ ); DTR, deep tendon reflexes, (areflexia 0, hyporeflexia 1, normal 2), NA not applicable. $\left({ }^{\#)}\right.$ approximately, Age at onset in years (y) CMAP compound muscle action potential $[\mathrm{mV}], S N A P$ sensory nerve action potential $[\mu \mathrm{V}], D L$ distal latency [ms], MNCV motor nerve conduction velocity [m/s], SCV sensory conduction velocity [m/s], NR no response. - none. Chromosomal position (GRCh37/hg19) 17:75488782, Reference sequence used: NM_006640.4

score of 23/30, and CERADplus scores indicating deficits in visual constructive abilities $(z=-3.71)$, and on the Trail Making Tests (Form A, $z=-1.71$; Form B, $z=-$ 2.34) (see also Fig. 1d). Like in his mother, his cranial MRI revealed no specific abnormality. His past medical history included substance abuse of tetrahydrocannabinol (THC).

Subject III.2, ("1979) second child of our index patient, was phenotypically healthy. No subclinical pathologies were found in either nerve conduction studies (Table 2) nor in the neuropsychological assessment (he scored 29/ 30 on the MOCA). Past medical history was unremarkable, besides past consumption of THC and temporary depressive disorder.

Unfortunately, additional family members were not available for clinical or genetic assessment. The family history, however, revealed more probably identically affected subjects: The index patient's father (I.2; "1928; †1975) as well as one older brother (II.1; *1953; †1992) had suffered from polyneuropathy with bilateral pes cavus and cognitive deficits; Remarkably, the index patient's twin brother (II.2; "1959) was reported to be phenotypically healthy. The index patient had two more children, one daughter (III.1; "1977), who had been diagnosed with early childhood cerebral palsy, and one further son (III.3; "1981), who was said to have no symptoms or signs of neuropathy.

\section{Genetic analysis}

Deletions or Duplications in PMP22, MFN2 and MPZ were excluded in our index case II.3. By targeted NGS and variant prioritization we identified a heterozygous single nucleotide change c.1406 $\mathrm{T}>\mathrm{C}$ in exon 8 of the SEPT9 gene (GRCh37/hg19 chr17:75488782) resulting in the amino acid change p.(Val469Ala) which - to our best knowledge - have not been reported in clinical context so far (Fig. 2a). According to the databases mentioned above (gnomAD and ExAC), MAF of the identified SEPT9 variant was very low $(0.02 \%)$ in the European (non-Finnish) population. The resulting p.(Val469Ala) substitution occurred at an evolutionarily conserved amino acid residue (Fig. 2b). In silico prediction analysis revealed pathogenicity in 2 out of 3 prediction tools (Table 1). The CADD score was 22.7 (CADD GRCh37-v1.4), indicating that the variant belongs to the top $1 \%$ of deleterious variants in the human genome [11]. In segregation analysis (subject III.2 and III.4) we found co-segregation of the distinct CMT1 phenotype, here III.4, with the SEPT9 variant. The variant was classified as "uncertain significance" (class 3, evidence of pathogenicity: PP1, PP2, PP3, PP4) based on ACMG guidelines [10].

\section{Discussion}

In this study, we used a targeted NGS approach to investigate the genetic cause of a distinct CMT1-phenotype in a German family. We identified a novel rare heterozygous SEPT9 variant, c.1406 T > C, (p.Val469Ala) in an evolutionary conserved region, predicted to be pathogenic. The variant was confirmed to co-segregate with the CMT-phenotype within the family.

Heterozygous SEPT9 mutations have been linked to hereditary neuralgic amyotrophy (HNA), a rare autosomal dominant recurrent focal neuropathy, clinically characterized by acute episodes of brachial plexus neuropathy with muscle weakness and atrophy, preceded by severe pain [5]. Interestingly, phenotypic heterogeneity in HNA has been well documented, such as persisting non-recurrent neuropathy symptoms and involvement of the lumbosacral plexus [13]. Besides this, additional clinical features, mainly dysmorphic symptoms and even bilateral pes cavus, have been found in individuals with HNA [14], which is a hallmark clinical symptom of 




CMT neuropathy. Both affected individuals in this study demonstrate this clinical feature (Fig. 1b/c).

Electrophysiological studies in HNA show predominantly axonal damages, however demyelination patterns, largely present in our family, have been detected in HNA likewise [15]. SEPT9 is highly expressed in glia cells, including Schwann cells which form the myelin sheet in neuronal tissues. Remarkably, a previous histological study demonstrated minor onion bulb formations, a neuropathological hallmark of CMT1, in an HNA patient, suggestive of Schwann cell alterations [16].

The ubiquitously expressed septin 9 (SEPT9) is one of the 13 members of septins, a highly conserved family of
GTP binding proteins that have been linked to a broad spectrum of cellular functions. Septins interact with the cytoskeleton, including actin, and function in processes such as cytokinesis, motility, and cell polarity. Other functions include angiogenesis and bacterial autophagy (for review see [6]). SEPT9 knockout in mice results in embryonic lethality [17]. Septins form oligomeric complexes, which can assemble into higher ordered structures such as filaments and rings. A well-known septin complex consists of alternating SEPT2/6/7/9-units [18].

The SEPT9 gene gives rise to at least eight different transcript variants generated by extensive alternative splicing of the N-terminal domain, which may have tissue-specific expression (Fig. 2c). However, all 
transcript variants maintain a highly conserved central region containing the polybasic domain $(\mathrm{PB})$ preceding a GTP-binding domain (G-domain) [19].

Interestingly, previously identified missense mutations in HNA patients - namely c. $262 \mathrm{C}>\mathrm{T}$, p.(Arg88Trp); c. $278 \mathrm{C}>\mathrm{T}$, p.(Ser93Phe); c.-131G > C (in 5'UTR) affect the N-terminal region. Hence, only a minority of septin9 isoforms are altered by these mutations [19] (Fig. 2c). In contrast, our identified variant c.1406 T > C, p.(Val469Ala), located in the C-terminal region, occurs in all known transcripts of SEPT9 (Fig. 2c). Therefore, a far more severe phenotype due to the involvement of multiple tissues, for instance the central nervous system (CNS), can be presumed.

To note, known genetic variants associated with cognitive impairment were not excluded in this family. However, due to similar cognitive deficits in the here identified SEPT9-variant carriers and the positive family history, cognitive deficits may be an additional clinical feature in the SEPT9-phenotype spectrum. Of interest, alterations of other members of the septin family have been associated with neurodegenerative disorders [20]: SEPT1-4 have been linked to Alzheimer's disease while for SEPT5 an association to Parkinson's disease has been reported [20]. Thus, one might speculate that the SEPT9 alteration could be associated with the cognitive changes in our current study. This hypothesis is further supported by the characteristic feature of septins to form oligomeric complexes [21]. Septin-septin interactions are formed by oligomerization of two neighbouring Gdomains. The identified Substitution p.(Val469Ala) in this study is situated within the functionally relevant (Gdomain) (Fig. 2c). By an alanine-for-valine substitution in this binding domain, essential protein-protein interactions and functions may be disturbed, particularly as valine unlike alanine contains two non-hydrogen substituents attached to their c-beta carbon [22]. Due to the location of p.(Val469Ala) in a central domain of the protein and the potential demolishing impact on oligomerisation we suppose a rather loss of function effect. Further studies are needed to confirm this hypothesis.

\section{Conclusions}

We identified a novel rare heterozygous SEPT9 gene variant in a family demonstrating a distinct CMT1 phenotype with overlapping clinical features to SEPT9 associated HNA and suggest a potential relevant functional implication on protein level. Thus, SEPT9 alterations may be considered as a genetic risk-factor in CMT1. However, future clinical and functional studies are needed to elucidate the pathogenic relevance.

\section{Abbreviations}

ACMG: American College of Medical Genetics; CADD: Combined Annotation Dependent Depletion; CERADplus: Consortium to Establish a Registry for
Alzheimer's Disease battery; CMAP: Compound muscle action potential; CMT: Charcot-Marie-Tooth disease; DL: Distal latency; DTR: Deep tendon reflexes; F: Female; HMSN: Hereditary motor and sensory neuropathy; HNA1: Hereditary neuralgic amyotrophy 1; LL: Lower limbs; M: Male; MNCV: Motor nerve conduction velocity; MOCA: Montreal cognitive assessment; MRI: Magnetic resonance imaging; NA: Not applicable; NR: No response; PB: Polybasic region; SCV: Sensory conduction velocity; SNAP: Sensory nerve action potential; THC: Tetrahydrocannabinol; UL: Upper limbs

\section{Acknowledgments}

We are grateful to the family for participating in this study.

\section{Authors' contributions}

GMG: conception, analyses, clinical evaluation, interpretation, drafting the manuscript; CB: genetic analyses, interpretation, revising the manuscript, BK: neuropsychological tests, analyses, interpretation, revising the manuscript, CS conception, analyses, interpretation, drafting the manuscript. AO: conception, genetic analyses, interpretation, drafting the manuscript. All authors read and approved the final manuscript.

\section{Funding}

GMG and AO were supported by PRACTIS - Clinician Scientist Program of Hannover Medical School, funded by the German Research Foundation (DFG, ME 3696/3-1).

\section{Availability of data and materials}

The identified variant in this study has been submitted to the ClinVar database at NCBI archives (ID: SCV001156521; https://www.ncbi.nlm.nih.gov/ clinvar/). All data generated or analysed during this study are included in this published article.

\section{Ethics approval and consent to participate}

The patients gave written consent for participating. The ethics committee at Hannover Medical School consented to the publication.

\section{Consent for publication}

The patients gave written consent for publishing.

\section{Competing interests}

The authors declare that they have no competing interests.

\section{Author details}

${ }^{1}$ Department of Neurology, Hannover Medical School, Carl-Neuberg-Str. 1, 30625 Hannover, Germany. ${ }^{2}$ Center for Genomics and Transcriptomics (CeGaT GmbH), Tübingen, Germany.

Received: 23 October 2019 Accepted: 25 February 2020

Published online: 02 March 2020

\section{References}

1. Werheid F, Azzedine H, Zwerenz E, Bozkurt A, Moeller MJ, Lin L, et al. Underestimated associated features in CMT neuropathies: clinical indicators for the causative gene? Brain Behav. 2016;6(4):e00451.

2. Mathis S, Goizet C, Tazir M, Magdelaine C, Lia A, Magy L, et al. CharcotMarie-Tooth diseases: an update and some new proposals for the classification. J Med Genet. 2015;52(10):681-90.

3. Saporta AS, Sottile SL, Miller LJ, Feely SM, Siskind CE, Shy ME. Charcot-MarieTooth disease subtypes and genetic testing strategies. Ann Neurol. 2011; 69(1):22-33

4. Azzedine H, Senderek J, Rivolta C, Chrast R. Molecular genetics of charcotmarie-tooth disease: from genes to genomes. Mol Syndromol. 2012;3(5): 204-14.

5. Kuhlenbaumer G, Hannibal MC, Nelis E, Schirmacher A, Verpoorten N, Meuleman J, et al. Mutations in SEPT9 cause hereditary neuralgic amyotrophy. Nat Genet. 2005;37(10):1044-6.

6. Mostowy S, Cossart P. Septins: the fourth component of the cytoskeleton. Nat Rev Mol Cell Biol. 2012;13(3):183-94.

7. Bai X, Bowen JR, Knox TK, Zhou K, Pendziwiat M, Kuhlenbaumer G, et al. Novel septin 9 repeat motifs altered in neuralgic amyotrophy bind and bundle microtubules. J Cell Biol. 2013;203(6):895-905. 
8. Nasreddine ZS, Phillips NA, Bedirian V, Charbonneau S, Whitehead V, Collin I, et al. The Montreal cognitive assessment, MoCA: a brief screening tool for mild cognitive impairment. J Am Geriatr Soc. 2005;53(4):695-9.

9. Ehrensperger MM, Berres M, Taylor KI, Monsch AU. Early detection of Alzheimer's disease with a total score of the German CERAD. J Int Neuropsychol Soc. 2010;16(5):910-20.

10. Richards S, Aziz N, Bale S, Bick D, Das S, Gastier-Foster J, et al. Standards and guidelines for the interpretation of sequence variants: a joint consensus recommendation of the American College of Medical Genetics and Genomics and the Association for Molecular Pathology. Genet Med. 2015; 17(5):405-24.

11. Kircher M, Witten DM, Jain P, O'Roak BJ, Cooper GM, Shendure J. A general framework for estimating the relative pathogenicity of human genetic variants. Nat Genet. 2014;46(3):310-5.

12. Lehrl S, Triebig G, Fischer B. Multiple choice vocabulary test MWT as a valid and short test to estimate premorbid intelligence. Acta Neurol Scand. 1995; 91(5):335-45.

13. van Alfen $N$, van Engelen BG. The clinical spectrum of neuralgic amyotrophy in 246 cases. Brain. 2006;129(Pt 2):438-50.

14. van Alfen N, van Engelen BG, Reinders JW, Kremer H, Gabreels FJ. The natural history of hereditary neuralgic amyotrophy in the Dutch population: two distinct types? Brain. 2000;123(Pt 4):718-23.

15. Ueda M, Kawamura N, Tateishi T, Sakae N, Motomura K, Ohyagi Y, et al. Phenotypic spectrum of hereditary neuralgic amyotrophy caused by the SEPT9 R88W mutation. J Neurol Neurosurg Psychiatry. 2010;81 (1):94-6.

16. Klein CJ, Dyck PJ, Friedenberg SM, Burns TM, Windebank AJ, Dyck PJ. Inflammation and neuropathic attacks in hereditary brachial plexus neuropathy. J Neurol Neurosurg Psychiatry. 2002;73(1):45-50.

17. Fuchtbauer A, Lassen LB, Jensen AB, Howard J, Quiroga Ade S, Warming $S$ et al. Septin9 is involved in septin filament formation and cellular stability. Biol Chem. 2011;392(8-9):769-77.

18. Estey MP, Kim MS, Trimble WS. Septins. Curr Biol. 2011;21(10):384.

19. Landsverk ML, Ruzzo EK, Mefford HC, Buysse K, Buchan JG, Eichler EE, et al. Duplication within the SEPT9 gene associated with a founder effect in north American families with hereditary neuralgic amyotrophy. Hum Mol Genet. 2009;18(7):1200-8.

20. Peterson EA, Petty EM. Conquering the complex world of human septins: implications for health and disease. Clin Genet. 2010;77(6):511-24.

21. Neubauer K, Zieger B. The mammalian septin interactome. Front Cell Dev Biol. 2017;5:3.

22. Betts MJ, Russell RB. Amino acid properties and consequences of substitutions. Bioinform Genet. 2003;317:289-316.

\section{Publisher's Note}

Springer Nature remains neutral with regard to jurisdictional claims in published maps and institutional affiliations.

Ready to submit your research? Choose BMC and benefit from:

- fast, convenient online submission

- thorough peer review by experienced researchers in your field

- rapid publication on acceptance

- support for research data, including large and complex data types

- gold Open Access which fosters wider collaboration and increased citations

- maximum visibility for your research: over $100 \mathrm{M}$ website views per year

At BMC, research is always in progress.

Learn more biomedcentral.com/submissions 